\title{
Nonresonant two-level transitions: Insights from quantum thermodynamics
}

\author{
Andreas Wacker (i) \\ Mathematical Physics and NanoLund, Lund University, Box 118, 22100 Lund, Sweden
}

(Received 27 September 2021; revised 11 November 2021; accepted 11 January 2022; published 27 January 2022)

\begin{abstract}
Based on concepts from quantum thermodynamics, the two-level system coupled to a single electromagnetic mode is analyzed. Focusing on the case of detuning, where the mode frequency does not match the transition frequency, effective energies are derived for the levels and the photon energy. It is shown that these should be used for energy exchange with fermionic and bosonic reservoirs in the steady state to achieve a thermodynamically consistent description. While recovering known features such as frequency pulling or Bloch gain, this sheds light on their thermodynamic background and allows for a coherent understanding.
\end{abstract}

DOI: 10.1103/PhysRevA.105.012214

\section{INTRODUCTION}

Two-level systems are the paradigm for the interaction of matter with light. Typically, one considers light frequencies $\omega / 2 \pi$, where the photon energy $\hbar \omega$ matches the energy difference $E_{u}-E_{l}$ between the upper (index $u$ ) and lower (index $l$ ) levels. However, it is well-known that optical transitions are broadened due to the finite lifetimes of levels and photons, so a certain width of frequencies can be emitted or absorbed. A straightforward question is how energy balance is satisfied for a finite detuning $\hbar \Delta=\hbar \omega+E_{l}-E_{u}$.

Detuning is known to have a variety of practical consequences, e.g., it results in frequency pulling [1] for lasers and Bloch gain for intersubband transitions in semiconductor heterostructures [2,3]. From a more fundamental point of view, there had been discussions on the thermodynamic consistency [4] for the archetypal Scovil\&Schulz-DuBois maser [5]. Detuned transitions also play an important role for certain gate operations on qubits in quantum information [6,7].

Here, the issue of detuning is studied from a quantumthermodynamic [8-11] perspective. Assuming local couplings [12-14] of the two levels with separate reservoirs, the energy balance in the steady state allows us to identify effective energies for the levels and the electromagnetic mode. These differ from the bare energies by a fraction of the detuning which is proportional to the contribution to the total broadening, see Eqs. (14) and (21) for the classical and quantum treatments, respectively. Applying the effective energies for the reservoir transitions, it is shown that both the first and second law of thermodynamics are satisfied.

In this paper, fermionic systems are considered, where the upper (lower) level is connected to a specific reservoir with

\footnotetext{
*Andreas.Wacker@teorfys.lu.se
}

Published by the American Physical Society under the terms of the Creative Commons Attribution 4.0 International license. Further distribution of this work must maintain attribution to the author(s) and the published article's title, journal citation, and DOI. Funded by Bibsam. electrochemical potential $\mu_{u / l}$, as depicted in Fig. 1. Such a setup was recently realized experimentally with high conversion efficiency for microwaves [15]. It is a prototype model system for many important devices such as light emitting diodes (LEDs), semiconductor lasers, or semiconductor solar cells, where the upper and lowers levels correspond to conduction and valence band states, respectively. Related results for the states of an atom coupled to bosonic reservoirs have been presented in Ref. [16], restricting to a classical field, which resolved the issues addressed in Ref. [4].

This paper is organized as follows: Section II briefly summaries the general concepts from quantum thermodynamics applied. The heart of the paper is Sec. III, where the two-level system is carefully analyzed using detailed calculations presented in Appendices A and B for the classical and quantum treatment of the electromagnetic mode, respectively. Sections IV and V consider the frequency pulling of lasers and the Bloch gain for intersubband transitions. For these examples, it is shown that the effective energies introduced here provide the same features as detailed microscopic calculations performed before. Finally, Appendix $\mathrm{C}$ details how the effective energies can be generalized to arbitrary systems with fermionic baths.

\section{GENERAL THERMODYNAMIC POINT OF VIEW}

We consider the system (e.g., the two-level system) in connection with reservoirs using the common quantumthermodynamics treatment. Let $\dot{U}_{\alpha}$ be the energy flow from the reservoir $\alpha$ into the system and $\dot{U}_{\text {opt }}$ the energy flow from the optical field into the system. Energy conservation implies in the steady state:

$$
\sum_{\alpha} \dot{U}_{\alpha}+\dot{U}_{\mathrm{opt}}=0
$$

The entropy production in reservoir $\alpha$ reads

$$
\frac{d S_{\alpha}}{d t}=-\frac{\dot{U}_{\alpha}}{T_{\alpha}}+\dot{N}_{\alpha} \frac{\mu_{\alpha}}{T_{\alpha}},
$$

where $\dot{N}_{\alpha}$ is the particle transfer from the reservoir $\alpha$ into the system. Then the second law of thermodynamics in the 


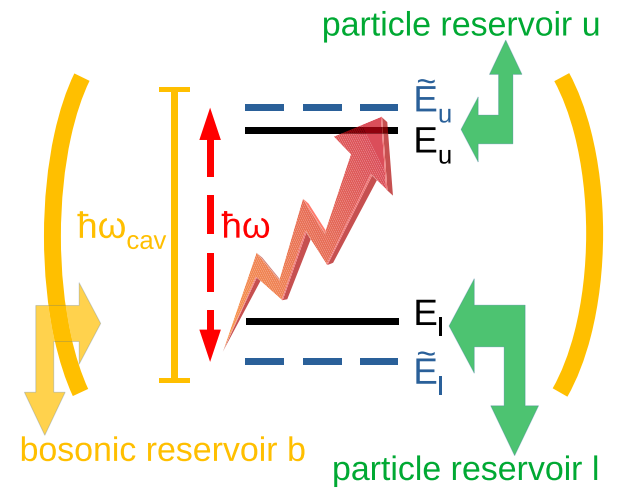

FIG. 1. Sketch of the two-level system (horizontal black full lines) coupled to particle reservoirs and a single mode electromagnetic field. If the photon energy $\hbar \omega$ does not match the energy difference between the levels, the analysis of energy fluxes provides effective energies (dashed blue horizontal lines), see Eqs. (14), which satisfy energy conservation $\tilde{E}_{u}-\tilde{E}_{l}=\hbar \omega$. For a quantum treatment of the cavity mode coupled to a bosonic reservoir, the light comes in portions with an effective energy $\tilde{E}_{\mathrm{ph}}=\hbar \omega$, see Eqs. (21) which differs from the photon energy of the empty cavity $\hbar \omega_{\text {cav }}$ by a fraction of the total detuning $\hbar \Delta_{\text {cav }}=\hbar \omega_{\text {cav }}+\tilde{E}_{l}-\tilde{E}_{u}$.

form of positive definite entropy production in the steady state requires

$$
\sum_{\alpha} \frac{d S_{\alpha}}{d t}+\frac{d S_{\mathrm{opt}}}{d t} \geqslant 0 .
$$

A standard quantum kinetic treatment is provided by the time evolution of the reduced density operator $\hat{\rho}$ of the system,

$$
\frac{d \hat{\rho}}{d t}=\frac{1}{\mathrm{i} \hbar}\left[\hat{H}_{S}, \hat{\rho}\right]+\sum_{\alpha} \mathcal{L}_{\alpha}[\hat{\rho}],
$$

where $\hat{H}_{S}$ the system Hamilton operator and $\mathcal{L}_{\alpha}$ describes the coupling to reservoir $\alpha$ within a Markovian treatment, see, e.g., Ref. [17]. Following well-established standard procedure [18-20], which is commonly used for the description of nanoscale engines, see, e.g., Ref. [21], the energy and particle flows from the reservoirs into the system are

$$
\dot{U}_{\alpha}=\operatorname{Tr}\left\{\hat{H}_{S} \mathcal{L}_{\alpha}[\hat{\rho}]\right\} \quad \text { and } \quad \dot{N}_{\alpha}=\operatorname{Tr}\left\{\hat{N}_{S} \mathcal{L}_{\alpha}[\hat{\rho}]\right\},
$$

respectively, where $\hat{N}_{S}$ is the number operator of the system. The corresponding power (work per time) transferred to the system reads

$$
P_{S}=\operatorname{Tr}\left\{\hat{\rho} \frac{\partial \hat{H}_{S}}{\partial t}\right\}
$$

which requires an absolute time-dependence of the Hamiltonian, as given by a classical optical field. It matches exactly the total rate of work $\boldsymbol{j} \cdot \mathcal{E}$ done by a classical electromagnetic field [22], where $\boldsymbol{j}$ is the current density and $\mathcal{E}$ is the electric field.

Such thermodynamic considerations have been frequently performed for the analysis of optoelectronic systems such as LEDs, lasers, and solar cells, see, e.g., Refs. [23,24] with a highlight on different aspects. The focus of this paper is to use Eqs. (1) and (3) as necessary conditions for the choice of $\mathcal{L}_{\alpha}$ in the construction of master equations. As shown below, this has distinct implications on the choice of energies used in the thermal occupation functions of the reservoirs for finite detuning between the optical modes and the two-level system.

In this paper, we stay within the realm of the quantum master Eq. (4) with Lindblad-type Liouvillians $\mathcal{L}_{\alpha}$, see Eq. (8), which is frequently applied to optical systems, see, e.g., Ref. [25]. For the case of classical fields with a timedependent Hamiltonian $\hat{H}_{S}(t)$, thermodynamic aspects of more detailed approaches have been studied in Refs. [26-28], where the impact of the Rabi splitting for strong classical fields is also addressed. It should be noted that the derivation of quantum master equations in the presence of time-dependent Hamiltonians $\hat{H}_{S}(t)$ is a matter of ongoing discussion, see, e.g., Ref. [29] and references cited therein.

\section{TWO-LEVEL SYSTEM WITH ELECTRONIC RESERVOIRS}

We consider an electronic two-level system with energies $E_{u}>E_{l}$ as given by the bare Hamiltonian

$$
\hat{H}_{0}=E_{u} \hat{c}_{u}^{\dagger} \hat{c}_{u}+E_{l} \hat{c}_{l}^{\dagger} \hat{c}_{l},
$$

which is coupled to electron reservoirs $\alpha \in\{u, l\}$ with temperatures $T_{\alpha}$ and electrochemical potential $\mu_{\alpha}$ via Lindblad operators $[30,31]$

$$
\begin{aligned}
\mathcal{L}_{\alpha}[\hat{\rho}] & =\gamma_{\alpha} f_{\alpha} \mathcal{D}_{\hat{c}_{\alpha}^{\dagger}}[\hat{\rho}]+\gamma_{\alpha}\left(1-f_{\alpha}\right) \mathcal{D}_{\hat{c}_{\alpha}}[\hat{\rho}] \\
\text { with } \quad \mathcal{D}_{\sigma}[\hat{\rho}] & =\sigma \hat{\rho} \sigma^{\dagger}-\frac{1}{2}\left(\sigma^{\dagger} \sigma \hat{\rho}+\hat{\rho} \sigma^{\dagger} \sigma\right),
\end{aligned}
$$

where $\gamma_{\alpha}$ denotes the coupling strength between level $\alpha$ and its connected reservoir. This is known as the local approach [12-14]. Commonly, one assumes that only the energy $E_{\alpha}$ of the isolated level is relevant for the transition to bath $\alpha$, which results in the occupation functions $f_{\alpha}=f_{\alpha}^{\text {common }}$ with the Fermi function

$$
f_{\alpha}^{\text {common }}=\frac{1}{\exp \left[\left(E_{\alpha}-\mu_{\alpha}\right) / k_{B} T_{\alpha}\right]+1} .
$$

However, it is known that such an approach can lead to violations of the thermodynamic rules $[4,12,32]$, which is also the case for the system studied here (see below). The key point of this paper is to trace such violations to the use of Eq. (9). Therefore, we keep the occupations $f_{\alpha}$ undefined until we can identify effective energies (14) and (21), which are suggested to replace the localized level energies $E_{\alpha}$ in Eq. (9). These effective energies reflect the coupling of the local levels to the other levels in the system (here by the light field), which is disregarded in the common use of the local approach.

Transitions between states $u$ and $l$ are possible by coupling to an optical field resulting in a net rate $R$ for transitions $u \rightarrow l$. Below, we provide detailed calculations of $R$ in the steady state (superscript ${ }^{\text {ss }}$ ) both for classical and quantum fields in Secs. III A and III B, respectively. In particular, we investigate the sign of $R^{\mathrm{ss}}$ obtained by quantum kinetics and compare it with the sign determined by thermodynamic considerations as outlined in Sec. II. 
Typically, such systems are treated under resonance $(\Delta=0)$ in the literature (see, e.g., Refs. $[33,34])$. Here the focus is on detuning with a finite value of $\Delta$.

\section{A. Classical field}

Within the common rotating wave approximation (RWA), we set $\hat{H}_{S}=\hat{H}_{0}+\hat{V}_{\mathrm{cl}}(t)$ with

$$
\hat{V}_{\mathrm{cl}}(t)=\hbar \epsilon \hat{c}_{u}^{\dagger} \hat{c}_{l} \mathrm{e}^{-i \omega t}+\hbar \epsilon^{*} \hat{c}_{l}^{\dagger} \hat{c}_{u} \mathrm{e}^{i \omega t},
$$

with the coupling strength $\epsilon$. A standard density matrix calculation, as detailed in Appendix A, provides the following results in the steady state, where $R^{\text {ss }}$ is the transition rate between the upper and lower level, as given by Eq. (A12): The net power absorbed from the electromagnetic field (6) is given by

$$
P_{s}^{\mathrm{ss}}=-\hbar \omega R^{\mathrm{ss}}
$$

and the particle and energy flows from the contacts (5) in the steady state read

$$
\begin{array}{ccc}
\dot{N}_{u}^{\mathrm{ss}}=R^{\mathrm{ss}} & \text { and } \quad \dot{N}_{l}^{\mathrm{ss}}=-R^{\mathrm{ss}}, \\
\dot{U}_{u}^{\mathrm{ss}}=\tilde{E}_{u} R^{\mathrm{ss}} & \text { and } \quad \dot{U}_{l}^{\mathrm{ss}}=-\tilde{E}_{l} R^{\mathrm{ss}},
\end{array}
$$

with the effective energies

$$
\tilde{E}_{u}=E_{u}+\frac{\gamma_{u} \hbar \Delta}{\gamma_{u}+\gamma_{l}} \quad \text { and } \quad \tilde{E}_{l}=E_{l}-\frac{\gamma_{l} \hbar \Delta}{\gamma_{u}+\gamma_{l}} .
$$

Note that this is a rigorous result for the system considered based on the general thermodynamic framework of Sec. II within the Markovian approximation and the local coupling to the reservoir (8). The effective energies can be interpreted as a distribution of the total detuning $\hbar \Delta$ to the bare levels according to their relative coupling strengths $\gamma_{\alpha}$, so they satisfy $\tilde{E}_{u}-\tilde{E}_{l}=\hbar \omega$. This reflects the fact that the sum of energy flows, $\dot{U}_{u}^{\text {ss }}+\dot{U}_{l}^{\text {ss }}+P_{S}=0$, conserves energy (1).

Based on these results, the entropy production (2) provides

$$
\dot{S}_{u}^{\mathrm{ss}}+\dot{S}_{l}^{\mathrm{ss}}=R^{\mathrm{ss}}\left(\frac{\tilde{E}_{l}-\mu_{l}}{T_{l}}-\frac{\tilde{E}_{u}-\mu_{u}}{T_{u}}\right) .
$$

Up to now, we performed all calculations without specifying the vales of $f_{\alpha}$, which need to reflect the reservoir properties. Now we use the criterion of positive definite entropy production (3) to identify these. According to Eq. (A12), $R^{\mathrm{ss}}$ has the same sign as $\left(f_{u}-f_{l}\right)$. Thus, positivity of entropy production (3) implies that $f_{u}-f_{l}$ needs to have the same sign as $\left(\frac{\tilde{E}_{l}-\mu_{l}}{T_{l}}-\frac{\widetilde{E}_{u}-\mu_{u}}{T_{u}}\right)$. This can be guaranteed if we choose

$$
f_{\alpha}=\frac{1}{\mathrm{e}^{\left(\tilde{E}_{\alpha}-\mu_{\alpha}\right) / k_{B} T_{\alpha}}+1}
$$

as $\left(\frac{1}{\mathrm{e}^{x_{u}}+1}-\frac{1}{\mathrm{e}^{x_{l}}+1}\right)$ has always the same sign as $\left(X_{l}-X_{u}\right)$ due to the strong monotonic decrease of $\frac{1}{\mathrm{e}^{x}+1}$. However, violations of Eq. (3) are possible if we choose the bare energy levels $E_{\alpha}$ in the bath Fermi functions following the tempting guess (9). We conclude that the effective energies (14) should be used for the occupation functions in the fermionic reservoirs in full analogy to the bosonic case treated in Ref. [16].

For equal temperatures, $T_{u}=T_{l}=T$, Eq. (15) shows that $R^{\mathrm{ss}}$ has the same sign as $\mu_{u}-\mu_{l}-\hbar \omega$. This implies operation as an LED if the bias $\mu_{u}-\mu_{l}$ surpasses the photon energy $\hbar \omega$ and operation as a solar cell in the opposite case. This is a consequence of the fact that no entropy is produced in the light field, which is described by a classical field in Eq. (10). Thus, the only source of entropy production is the generation of heat from the excess energy $\mu_{u}-\mu_{l}>\hbar \omega$ for emission or $\hbar \omega>\mu_{u}-\mu_{l}$ for absorption, which is transferred to the reservoirs.

Note, that this procedure also applies to tunneling problems, which correspond to $\omega=0$. Thereby it resolves the violation of the second law for the local approach in Ref. [12].

\section{B. Quantized field}

Within the RWA, we set in the spirit of the JaynesCummings model [35]

$$
\hat{V}_{\mathrm{JC}}=\hbar g \hat{c}_{u}^{\dagger} \hat{c}_{l} \hat{a}+\hbar g^{*} \hat{c}_{l}^{\dagger} \hat{c}_{u} \hat{a}^{\dagger}+\hbar \omega_{\mathrm{cav}} \hat{a}^{\dagger} \hat{a},
$$

with the bosonic annihilation operator $\hat{a}$ for the photon mode. Now the detuning is given by $\hbar \Delta_{\text {cav }}=\hbar \omega_{\text {cav }}+E_{l}-E_{u}$. To allow for a steady state, we add the interaction of the photon mode with a thermal bosonic reservoir with average occupation $n_{\mathrm{b}}$ and transition rate $\gamma_{\mathrm{b}}$ via the Lindblad operator

$$
\mathcal{L}_{\mathrm{b}}[\hat{\rho}]=\gamma_{\mathrm{b}}\left(n_{\mathrm{b}}+1\right) \mathcal{D}_{\hat{a}}[\hat{\rho}]+\gamma_{\mathrm{b}} n_{\mathrm{b}} \mathcal{D}_{\hat{a}^{\dagger}}[\hat{\rho}] .
$$

As for the fermionic occupation factors $f_{\alpha}$, the value of $n_{b}$ is specified at a later stage. This provides the equation of motion for the density operator:

$$
\frac{d \hat{\rho}}{d t}=\frac{1}{i \hbar}\left[\hat{H}_{0}+\hat{V}_{\mathrm{JC}}, \hat{\rho}\right]+\mathcal{L}_{u}[\hat{\rho}]+\mathcal{L}_{l}[\hat{\rho}]+\mathcal{L}_{\mathrm{b}}[\hat{\rho}] .
$$

As described in Appendix B, this provides the following results in the steady state with the net transition rate $R^{\mathrm{ss}}$, for which we do not have a closed expression:

$$
\begin{array}{r}
\dot{N}_{u}^{\mathrm{ss}}=R^{\mathrm{ss}} \quad \text { and } \quad \dot{N}_{l}^{\mathrm{ss}}=-R^{\mathrm{ss}}, \\
\dot{U}_{u}^{\mathrm{ss}}=\tilde{E}_{u} R^{\mathrm{ss}}, \quad \dot{U}_{l}^{\mathrm{ss}}=-\tilde{E}_{l} R^{\mathrm{ss}}, \\
\text { and } \quad \dot{U}_{\mathrm{b}}^{\mathrm{ss}}=-\tilde{E}_{\mathrm{ph}} R^{\mathrm{ss}}
\end{array}
$$

with the effective energies

$$
\begin{aligned}
\tilde{E}_{u} & =E_{u}+\frac{\gamma_{u} \hbar \Delta_{\mathrm{cav}}}{\gamma_{u}+\gamma_{l}+\gamma_{\mathrm{b}}}, \\
\tilde{E}_{l} & =E_{l}-\frac{\gamma_{l} \hbar \Delta_{\mathrm{cav}}}{\gamma_{u}+\gamma_{l}+\gamma_{\mathrm{b}}}, \\
\text { and } \quad \tilde{E}_{\mathrm{ph}} & =\hbar \omega_{\mathrm{cav}}-\frac{\gamma_{\mathrm{b}} \hbar \Delta_{\mathrm{cav}}}{\gamma_{u}+\gamma_{l}+\gamma_{\mathrm{b}}},
\end{aligned}
$$

which satisfy $\tilde{E}_{u}=\tilde{E}_{l}+\tilde{E}_{\mathrm{ph}}$, so the fluxes (20) fulfill energy conservation (1). This is analogous to Eqs. (14) for the classical treatment. However, the finite lifetime of the photon mode in the cavity contributes now to the broadening and, correspondingly, we obtain an effective photon energy $\tilde{E}_{\mathrm{ph}}$, which is relevant for the transitions with the bosonic bath.

Now we consider the requirement for positive entropy production (3). Applying the energy and particle currents above and Eq. (2), the entropy condition reads

$$
R^{\mathrm{ss}}\left[\frac{\tilde{E}_{\mathrm{ph}}}{T_{\mathrm{b}}}+\frac{\tilde{E}_{l}-\mu_{l}}{T_{l}}-\frac{\tilde{E}_{u}-\mu_{u}}{T_{u}}\right] \geqslant 0,
$$


where $T_{\mathrm{b}}$ is the temperature of the bosonic bath coupled to the photon mode.

In Appendix B, it is shown within a Hartree-Fock like approximation, that

the sign of $R^{\mathrm{ss}}$ equals the sign of $\frac{f_{u}}{1-f_{u}}-\frac{f_{l}}{1-f_{l}} \times \frac{n_{\mathrm{b}}}{1+n_{\mathrm{b}}}$.

Applying the Fermi and Bose distributions

$$
\begin{aligned}
f_{u} & =\frac{1}{\mathrm{e}^{\left(\tilde{E}_{u}-\mu_{u}\right) / k_{B} T_{u}}+1}, \quad f_{l}=\frac{1}{\mathrm{e}^{\left(\tilde{E}_{l}-\mu_{l}\right) / k_{B} T_{l}}+1}, \\
\text { and } n_{\mathrm{ph}} & =\frac{1}{\mathrm{e}^{\tilde{E}_{\mathrm{ph}} / k_{B} T_{\mathrm{b}}}-1},
\end{aligned}
$$

Eq. (23) becomes

$$
\begin{aligned}
& \mathrm{e}^{-\left(\tilde{E}_{u}-\mu_{u}\right) / k_{B} T_{u}}-\mathrm{e}^{-\tilde{E}_{\mathrm{ph}} / k_{B} T_{\mathrm{b}}} \times \mathrm{e}^{-\left(\tilde{E}_{l}-\mu_{l}\right) / k_{\mathrm{b}} T_{l}} \\
& \text { determines the sign of } R^{\mathrm{ss}},
\end{aligned}
$$

which guaranties the positivity in Eq. (22) due to the monotonic increase of the exponential function. Here it is crucial that the effective energies (21) enter the respective occupation functions. Otherwise, violations of Eq. (22) can be easily constructed for particular values of temperatures and electrochemical potentials.

For equal temperatures, $T_{u}=T_{l}=T$, Eq. (22) shows that $R^{\mathrm{ss}}$ has the same sign as $\mu_{u}-\mu_{l}-\tilde{E}_{\mathrm{ph}}\left(1-T / T_{\mathrm{b}}\right)$. Thus, light emission $\left(R^{\mathrm{ss}}>0\right)$ is even possible for $\mu_{u}-\mu_{l}<\tilde{E}_{\mathrm{ph}}$, provided that $T_{\mathrm{b}}$ is not too large. In this case, the LED emits more light than it consumes electrical energy, which has been experimentally verified [36]. The excess energy is taken from the reservoirs which provide cooling [37]. For operation as a solar cell $\left(R^{\mathrm{ss}}<0\right)$, this shows that the extracted electrical power $P_{\mathrm{el}}=-R^{\mathrm{ss}}\left(\mu_{u}-\mu_{l}\right)$ is limited by

$$
P_{\mathrm{el}} \leqslant \dot{U}_{\mathrm{b}}^{\mathrm{ss}} \frac{T_{\mathrm{b}}-T}{T_{\mathrm{b}}}
$$

which is just Carnot's law for the incoming heat $\dot{U}_{\mathrm{b}}^{\text {ss }}$ from the warm reservoir with $T_{\mathrm{b}}>T$.

\section{RELATION TO FREQUENCY PULLING IN A LASER}

For laser operation, the coherent states are a better approximation for the optical field than number states [38]. Technically, this can be done by the semiclassical approximation [39], where $\langle\hat{a}\rangle$ is treated as a classical field. Therefore, in Eqs. (B1) and (B2), the approximation

$$
Y=g^{*} \operatorname{Tr}\left\{\hat{c}_{l}^{\dagger} \hat{c}_{u} \hat{a}^{\dagger} \hat{\rho}\right\} \approx g^{*} a^{*} \sigma_{u l}
$$

is used with

$$
\sigma_{u l}=e^{i \omega t} \operatorname{Tr}\left\{\hat{c}_{l}^{\dagger} \hat{c}_{u} \hat{\rho}\right\} \quad \text { and } \quad a=e^{i \omega t} \operatorname{Tr}\{\hat{a} \hat{\rho}\},
$$

where a natural oscillation with frequency $\omega$ is assumed. Without detuning, $\omega=\omega_{\text {cav }}=\left(E_{u}-E_{l}\right) / \hbar$ is the natural choice, which corresponds to the interaction picture. For the case of detuning, $\omega$ is determined below. Equation (18) provides

$$
\begin{gathered}
\frac{d}{d t} \sigma_{u l}=i\left(\omega-\frac{E_{u}-E_{l}}{\hbar}\right) \sigma_{u l}+i g a\left(\sigma_{u u}-\sigma_{l l}\right) \\
-\frac{\gamma_{u}+\gamma_{l}}{2} \sigma_{u l}, \\
\frac{d}{d t} a=i\left(\omega-\omega_{\text {cav }}\right) a-i g^{*} \sigma_{u l}-\frac{\gamma_{\mathrm{b}}}{2} a,
\end{gathered}
$$

which, together with (B1) and (B2), give a closed set of equations. In the steady state, Eq. (26) provides

$$
\sigma_{u l}^{\mathrm{ss}}=\frac{\omega-\omega_{\mathrm{cav}}+i \gamma_{\mathrm{b}} / 2}{|g|^{2}} g a^{\mathrm{ss}} .
$$

With the semiclassical approximation (24), the set of equations for the quantum case (B1), (B2), and (25) equal the classical treatment, as given in Eqs. (A1), (A2), and (A3), with $\epsilon=g a$. Thus, we obtain from Eqs. (A8) and (A11)

$$
\sigma_{u l}^{\mathrm{ss}}=\frac{-H\left(\left|g a^{\mathrm{ss}}\right|^{2}\right)\left(f_{u}-f_{l}\right)}{\Delta+i\left(\gamma_{u}+\gamma_{l}\right) / 2} g a^{\mathrm{ss}} .
$$

Equations (27) and (28) are only consistent if

$$
\begin{aligned}
(\omega & \left.-\omega_{\mathrm{cav}}+i \gamma_{\mathrm{b}} / 2\right)\left[\Delta+i\left(\gamma_{u}+\gamma_{l}\right) / 2\right] \\
= & -|g|^{2} H\left(\left|g a^{\mathrm{ss}}\right|^{2}\right)\left(f_{u}-f_{l}\right) .
\end{aligned}
$$

While the real part of the equation provides the field strength $a^{\text {ss }}$ of laser light in the cavity due to gain saturation, its imaginary part determines $\omega$ : As the right-hand side is purely real, the imaginary part needs to be zero on the left-hand side. Using $\Delta=\omega-\left(E_{u}-E_{l}\right) / \hbar$, one obtains

$$
\hbar \omega=\frac{\left(\gamma_{u}+\gamma_{l}\right) \hbar \omega_{\mathrm{cav}}+\gamma_{\mathrm{b}}\left(E_{u}-E_{l}\right)}{\gamma_{u}+\gamma_{l}+\gamma_{\mathrm{b}}} .
$$

Straightforward algebra shows that $\hbar \omega=\tilde{E}_{\text {ph }}$ from Eqs. (21). Thus, the effective photon energy obtained in Sec. III B matches the actual oscillation frequency in the laser. Furthermore, the effective energies $\tilde{E}_{u}$ and $\tilde{E}_{l}$ from the classical field (14) and the quantum treatment (21) agree with each other for this choice of $\hbar \omega$.

Equation (30) shows that for detuning between the cavity frequency and the optical transition frequency, the laser operates at a frequency in between. This is known as frequency pulling [1]. Actually, the expression (12.23) of Ref. [1] is directly obtained from Eq. (30) by introducing the $Q$ factors $Q_{c}=\omega / \gamma_{\mathrm{b}}$ and $Q_{a}=\omega /\left(\gamma_{u}+\gamma_{l}\right)$ for the cavity and the atomic transition, respectively. Thus, the treatment by heat flows suggested here provides the same result as a detailed optoelectronic study.

\section{RELATION TO BLOCH GAIN}

For layered semiconductor structures, optical transitions occur between subbands with a well-defined transition energy $E_{u}-E_{l}$ due to the quantized energies in the growth direction [40]. In addition, the lateral free-particle motion (with energy $E_{k}$ described by wave vector $k$ ) provides a continuous degree of freedom with occupation functions $f_{u}\left(E_{k}\right)$ and $f_{l}\left(E_{k}\right)$ in each of the subbands. Due to intrasubband scattering, the levels are broadened and thus light emission (absorption) is possible for detuning, with 
a finite value of $\Delta=\omega-\left(E_{u}-E_{l}\right) / \hbar$. A microscocopic density-matrix approach for the scattering [2] provided the steady-state net transition rate between the upper and lower subbands,

$$
R_{\mathrm{DM}}^{\mathrm{ss}}\left(k_{0}\right) \propto \frac{\gamma_{u}+\gamma_{l}}{\Delta^{2}+\left(\gamma_{u}+\gamma_{l}\right)^{2} / 4}\left\{\frac{\gamma_{l}\left[f_{u}\left(E_{k_{0}}\right)-f_{l}\left(E_{k_{0}}-\hbar \Delta\right)\right]}{\gamma_{u}+\gamma_{l}}+\frac{\gamma_{u}\left[f_{u}\left(E_{k_{0}}+\hbar \Delta\right)-f_{l}\left(E_{k_{0}}\right)\right]}{\gamma_{u}+\gamma_{l}}\right\},
$$

for states with a particular value of $k=k_{0}$ (which is essentially conserved in the optical transition); see Eq. (20) of Ref. [2]. The first factor provides the common Lorentzian broadening of the line and the second factor shows that the transitions are driven by differences between the occupations of the subbands. This factor is not just $f_{u}\left(E_{k_{0}}\right)-f_{l}\left(E_{k_{0}}\right)$ as frequently assumed, but has different energy arguments. This leads to a particular gain spectrum for the case of equal occupation of both subbands $\left[f_{u}(E)=f_{l}(E)\right]$, called dispersive gain or Bloch gain due to its relation to Bloch oscillations in superlattices [41,42]. This type of gain could be observed in quantum cascade lasers [3] and has been recently suggested to be relevant for the generation of frequency combs [43].

Analyzing the occupation factors of Eq. (31) reveals that the occupations of the upper level are taken at an average energy $\tilde{E}_{k_{0}}^{u}=E_{k_{0}}+\hbar \Delta \gamma_{u} /\left(\gamma_{u}+\gamma_{l}\right)$. Correspondingly, the occupations of the lower subband are taken at an average energy $\tilde{E}_{k_{0}}^{l}=E_{k_{0}}-\hbar \Delta \gamma_{l} /\left(\gamma_{u}+\gamma_{l}\right)$, so $E_{u}+\tilde{E}_{k_{0}}^{u}-\left(E_{l}+\right.$ $\left.\tilde{E}_{k_{0}}^{l}\right)=\hbar \omega$. This fully corresponds to the choice (14) obtained above, which therefore provides the same characteristic Bloch gain. The average energies (14) can alternatively be obtained as average transition energies within a Green's function treatment [16] (see Ref. [44] for the connection with Bloch gain).

\section{CONCLUSION}

Applying a quantum thermodynamic approach, effective energies were identified to describe optical transition under detuning, as given in Eqs. (14) and (21) for the classical and quantum treatments, respectively. These effective energies satisfy energy conservation by distributing the detuning to the bare energies according to their respective contribution to the broadening of the transition. Applying these effective energies for transitions with thermal reservoirs provides thermodynamic consistency within the generally accepted heat and work definitions (5), (6), where both the first and second laws are satisfied for steady-state operation.

The effective energy of the photon could be attributed to the pulled frequency in a laser cavity. Similarly, the effective level energies agree with the average energies associated with inter-subband transitions. Thus, the approach used here provides a comprehensive view of features obtained from different sophisticated microscopic treatments.

All results obtained are rigorous within the Markovian Lindblad master equation used. The only exception is the fulfillment of the second law for the quantum treatment of the cavity mode, where a Hartree-Fock-like approximation was required. It would be interesting how far the results can be generalized. Another open issue is whether these effective energies can also be applied for noise calculations, where different types of averages apply.
It is interesting to note that the effective energies appear independently of whether the coupling to the electromagnetic field is treated classically by a time-dependent Hamiltonian and quantum mechanically within a time-independent Hamiltonian. Thus, these findings appear not to be affected by the question in how far the quantum master equation Eq. (4) is applicable for time-dependent Hamiltonians.

For the classical case, the results can be generalized to arbitrary fermionic systems with a time-periodic Hamiltonian, see Appendix C. However, the identification of the effective energies then relies on a self-consistent solution of the system dynamics with the occupation functions, which may limit practical applications. (The recently developed thermodynamically consistent local approach [45] is an interesting alternative at the cost of a limited resolution for heat.) Albeit, the approach is thermodynamically consistent for arbitrary system parameters, one has to remember that the local approach (8) restricts to a single transition energy, which does not allow the detailed description of photon-assisted reservoir transitions or Coulomb blockade phenomena.

\section{ACKNOWLEDGMENTS}

The author thanks Alex Kalaee, Patrick Potts, and Peter Samuelsson for helpful discussions and collaboration on related issues. Financial support from the Knut and Alice Wallenberg Foundation (Project No. 2016.0089), the Swedish Research Council (Project No. 2017-04287), and NanoLund is gratefully acknowledged.

\section{APPENDIX A: CALCULATIONS FOR THE CLASSICAL CASE}

The quantum master equation (4) with $\hat{H}_{S}=\hat{H}_{0}+\hat{V}_{\mathrm{cl}}(t)$ based on the operators (7) and (10) can be mapped to a time-independent problem in the rotating frame where $\hat{A}^{R}=$ $\hat{U}(t) \hat{A} \hat{U}^{\dagger}(t)$, with $\hat{U}(t)=e^{i\left[\left(E_{l}+\hbar \omega\right) \hat{c}_{u} \hat{c}_{u}+E_{l} \hat{c}_{l} \hat{c}_{l}\right] t / \hbar}$, and provides the equation of motion for the density operator

$$
\begin{aligned}
\frac{d \hat{\rho}^{R}}{d t}= & -i\left[-\Delta \hat{c}_{u}^{\dagger} \hat{c}_{u}+\epsilon \hat{c}_{u}^{\dagger} \hat{c}_{l}+\epsilon^{*} \hat{c}_{l}^{\dagger} \hat{c}_{u}, \hat{\rho}^{R}\right] \\
& +\mathcal{L}_{u}\left[\hat{\rho}^{R}\right]+\mathcal{L}_{l}\left[\hat{\rho}^{R}\right],
\end{aligned}
$$

with $\Delta=\omega-\left(E_{u}-E_{l}\right) / \hbar$. This gives the equations of motion for the reduced density matrix $\sigma_{i j}=\operatorname{Tr}\left\{\hat{c}_{j}^{\dagger} \hat{c}_{i} \hat{\rho}^{\mathrm{R}}\right\}$ :

$$
\begin{gathered}
\frac{d}{d t} \sigma_{u u}=\gamma_{u}\left(f_{u}-\sigma_{u u}\right)+i\left(\epsilon^{*} \sigma_{u l}-\epsilon \sigma_{l u}\right), \\
\frac{d}{d t} \sigma_{l l}=\gamma_{l}\left(f_{l}-\sigma_{l l}\right)+i\left(\epsilon \sigma_{l u}-\epsilon^{*} \sigma_{u l}\right), \\
\frac{d}{d t} \sigma_{u l}=i \Delta \sigma_{u l}+i \epsilon\left(\sigma_{u u}-\sigma_{l l}\right)-\frac{\gamma_{u}+\gamma_{l}}{2} \sigma_{u l} .
\end{gathered}
$$


From the changes of the populations, we identify the net transition rate $u \rightarrow l$ :

$$
R=-i\left(\epsilon^{*} \sigma_{u l}-\epsilon \sigma_{u l}^{*}\right)=2 \operatorname{Im}\left\{\epsilon^{*} \sigma_{u l}\right\} .
$$

Furthermore, the work flow (6) becomes

$$
\begin{aligned}
P_{S} & =\operatorname{Tr}\left\{i \hbar \omega\left(\epsilon^{*} \hat{c}_{l}^{\dagger} \hat{c}_{u} e^{i \omega t}-\epsilon \hat{c}_{u}^{\dagger} \hat{c}_{l} e^{-i \omega t}\right) \hat{\rho}\right\} \\
& =i \hbar \omega \operatorname{Tr}\left\{\left(\epsilon^{*} \hat{c}_{l}^{\dagger} \hat{c}_{u}-\epsilon \hat{c}_{u}^{\dagger} \hat{c}_{l}\right) \hat{\rho}^{R}\right\}=-\hbar \omega R .
\end{aligned}
$$

This shows that each transition from the upper to lower level is associated with the energy portion $\hbar \omega$ removed from the system even if $E_{u}-E_{l} \neq \hbar \omega$. This agrees with the concept that the transition is associated with the creation of one photon with angular frequency $\omega$, albeit the optical field is treated as a classical variable in Eq. (10). In the steady state, Eq. (A5) directly provides Eq. (11).

The energy and particle flows from the leads (5) are

$$
\begin{aligned}
\dot{U}_{\alpha} & =\operatorname{Tr}\left\{\hat{H} \mathcal{L}_{\alpha}[\hat{\rho}]\right\}=\operatorname{Tr}\left\{\hat{H}^{R} \mathcal{L}_{\alpha}\left[\hat{\rho}^{R}\right]\right\} \\
& =E_{\alpha} \gamma_{\alpha}\left(f_{\alpha}-\sigma_{\alpha \alpha}\right)-\frac{\hbar \gamma_{\alpha}}{2}\left(\epsilon \sigma_{l u}+\epsilon^{*} \sigma_{u l}\right)
\end{aligned}
$$

and

$$
\dot{N}_{\alpha}=\operatorname{Tr}\left\{\left(\hat{c}_{u}^{\dagger} \hat{c}_{u}+\hat{c}_{l}^{\dagger} \hat{c}_{l}\right) \mathcal{L}_{\alpha}[\hat{\rho}]\right\}=\gamma_{\alpha}\left(f_{\alpha}-\sigma_{\alpha \alpha}\right) .
$$

In the steady state, Eq. (A3) provides

$$
\sigma_{u l}^{\mathrm{ss}}=\frac{-\epsilon\left(\sigma_{u u}-\sigma_{l l}\right)}{\Delta+i\left(\gamma_{u}+\gamma_{l}\right) / 2}
$$

Thus, we can relate the transition rate (A4) to the real part of $\epsilon^{*} \sigma_{u l}^{\mathrm{ss}}$ by

$$
\operatorname{Re}\left\{\epsilon^{*} \sigma_{u l}^{\mathrm{ss}}\right\}=-\frac{2 \Delta}{\gamma_{u}+\gamma_{l}} \operatorname{Im}\left\{\epsilon^{*} \sigma_{u l}^{\mathrm{ss}}\right\}=-\frac{\Delta}{\gamma_{u}+\gamma_{l}} R^{\mathrm{ss}} .
$$

In the steady state, Eqs. (A1) and (A2) become

$$
\gamma_{u}\left(f_{u}-\sigma_{u u}\right)=R^{\mathrm{ss}} \text { and } \gamma_{l}\left(f_{l}-\sigma_{l l}\right)=-R^{\mathrm{ss}}
$$

and thus Eq. (A7) provides Eqs. (12) of the main text. Inserting Eqs. (A9) and (A10) into the energy flows (A6) from the leads provides Eqs. (13) and (14), which are the key results.

Eqs. (A4) and (A8) provide the steady-state transition rate:

$$
R^{\mathrm{ss}}=\alpha\left(\sigma_{u u}-\sigma_{l l}\right), \quad \text { with } \quad \alpha=\frac{|\epsilon|^{2}\left(\gamma_{u}+\gamma_{l}\right)}{\left(\gamma_{u}+\gamma_{l}\right)^{2} / 4+\Delta^{2}} .
$$

Then, Eqs. (A1) and (A2) result in

$$
\left(\begin{array}{cc}
\gamma_{u}+\alpha & -\alpha \\
-\alpha & \gamma_{l}+\alpha
\end{array}\right)\left(\begin{array}{c}
\sigma_{u u}^{\mathrm{ss}} \\
\sigma_{l l}^{\mathrm{ss}}
\end{array}\right)=\left(\begin{array}{l}
\gamma_{u} f_{u} \\
\gamma_{l} f_{l}
\end{array}\right) \Rightarrow\left(\begin{array}{c}
\sigma_{u u}^{\mathrm{ss}} \\
\sigma_{l l}^{\mathrm{ss}}
\end{array}\right)=\frac{1}{\gamma_{u} \gamma_{l}+\alpha\left(\gamma_{u}+\gamma_{l}\right)}\left(\begin{array}{c}
\alpha\left(\gamma_{u} f_{u}+\gamma_{l} f_{l}\right)+\gamma_{u} \gamma_{l} f_{u} \\
\alpha\left(\gamma_{u} f_{u}+\gamma_{l} f_{l}\right)+\gamma_{u} \gamma_{l} f_{l}
\end{array}\right),
$$

so

$$
\sigma_{u u}^{\mathrm{ss}}-\sigma_{l l}^{\mathrm{ss}}=H\left(|\epsilon|^{2}\right)\left(f_{u}-f_{l}\right) \quad \text { with } \quad H\left(|\epsilon|^{2}\right)=\frac{\gamma_{u} \gamma_{l}}{\gamma_{u} \gamma_{l}+\alpha\left(\gamma_{u}+\gamma_{l}\right)}=\frac{1}{1+|\epsilon|^{2} \frac{\left(\gamma_{u}+\gamma_{l}\right)^{2}}{\gamma_{u} \gamma_{l}\left[\left(\gamma_{u}+\gamma_{l}\right)^{2} / 4+\Delta^{2}\right]}}
$$

and we find

$$
R^{\mathrm{ss}}=\alpha H\left(f_{u}-f_{l}\right),
$$

with positive $\alpha$ and $H$.

\section{APPENDIX B: CALCULATIONS THE QUANTUM CASE}

Defining the average occupation of the photon mode $n_{\mathrm{ph}}=$ $\operatorname{Tr}\left\{\hat{a}^{\dagger} \hat{a} \hat{\rho}\right\}$ and $Y=g^{*} \operatorname{Tr}\left\{\hat{c}_{l}^{\dagger} \hat{c}_{u} \hat{a}^{\dagger} \hat{\rho}\right\}$, Eq. (18) provides the equations of motion

$$
\begin{gathered}
\frac{d}{d t} \sigma_{u u}=\gamma_{u}\left(f_{u}-\sigma_{u u}\right)+i\left(Y-Y^{*}\right), \\
\frac{d}{d t} \sigma_{l l}=\gamma_{l}\left(f_{l}-\sigma_{l l}\right)-i\left(Y-Y^{*}\right), \\
\frac{d}{d t} n_{\mathrm{ph}}=\gamma_{\mathrm{b}}\left(n_{\mathrm{b}}-n_{\mathrm{ph}}\right)-i\left(Y-Y^{*}\right), \\
\frac{d}{d t} Y=i \Delta_{\mathrm{cav}} Y+i|g|^{2} F-\frac{\gamma_{u}+\gamma_{l}+\gamma_{\mathrm{b}}}{2} Y, \\
\text { with } F=\operatorname{Tr}\left\{\hat{c}_{u}^{\dagger} \hat{c}_{u}\left(1-\hat{c}_{l}^{\dagger} \hat{c}_{l}\right) \hat{\rho}\right\} \\
+\operatorname{Tr}\left\{\left(\hat{c}_{u}^{\dagger} \hat{c}_{u}-\hat{c}_{l}^{\dagger} \hat{c}_{l}\right) \hat{a}^{\dagger} \hat{a} \hat{\rho}\right\},
\end{gathered}
$$

where $\Delta_{\text {cav }}=\omega_{\text {cav }}-\left(E_{u}-E_{l}\right) / \hbar$. Equations (B1)-(B3) have a very transparent interpretation: The occupations of the levels and the photon mode are fed (emptied) by the respective reservoirs with corresponding rates $\gamma_{\alpha}$, where the speed is proportional to the occupation differences. In addition, there are optical transitions $u \rightarrow l$ generating photons with a rate

$$
R=2 \operatorname{Im}\{Y\} \text {. }
$$

The new quantity $Y$ satisfies a linear differential equation, where the inhomogeneity is determined by the expression $F$ related to spontaneous $\left[\propto \operatorname{Tr}\left\{\hat{c}_{u}^{\dagger} \hat{c}_{u}\left(1-\hat{c}_{l}^{\dagger} \hat{c}_{l}\right) \hat{\rho}\right\}\right]$ and stimulated $\left[\propto \operatorname{Tr}\left\{\left(\hat{c}_{u}^{\dagger} \hat{c}_{u}-\hat{c}_{l}^{\dagger} \hat{c}_{l}\right) \hat{a}^{\dagger} \hat{a} \hat{\rho}\right\}\right]$ emission. These provide further dynamical variables, so we do not have a closed system of equations.

The energy flows from the fermionic reservoirs (5) are

$$
\begin{aligned}
\dot{U}_{\alpha} & =\operatorname{Tr}\left\{\hat{H}_{S} \mathcal{L}_{\alpha}[\hat{\rho}]\right\} \\
& =E_{\alpha} \gamma_{\alpha}\left(f_{\alpha}-\sigma_{\alpha \alpha}\right)-\frac{\hbar \gamma_{\alpha}}{2}\left(Y+Y^{*}\right)
\end{aligned}
$$

and the corresponding energy flow from the bosonic reservoir is

$$
\begin{aligned}
\dot{U}_{\mathrm{b}} & =\operatorname{Tr}\left\{\hat{H}_{S} \mathcal{L}_{\mathrm{b}}[\hat{\rho}]\right\} \\
& =\hbar \omega_{\mathrm{cav}} \gamma_{\mathrm{b}}\left(n_{\mathrm{b}}-n_{\mathrm{ph}}\right)-\frac{\hbar \gamma_{\mathrm{b}}}{2}\left(Y+Y^{*}\right) .
\end{aligned}
$$

Let us now consider the steady state. Then, Eq. (B4) provides

$$
Y^{\mathrm{ss}}=-\frac{|g|^{2} F}{\Delta_{\mathrm{cav}}+i \frac{\gamma_{u}+\gamma_{l}+\gamma_{\mathrm{b}}}{2}},
$$


which implies

$$
\operatorname{Re}\left\{Y^{\mathrm{ss}}\right\}=-\frac{2 \Delta_{\mathrm{cav}} \operatorname{Im}\left\{Y^{\mathrm{ss}}\right\}}{\gamma_{u}+\gamma_{l}+\gamma_{\mathrm{b}}}=-\frac{\Delta_{\mathrm{cav}} R^{\mathrm{ss}}}{\gamma_{u}+\gamma_{l}+\gamma_{\mathrm{b}}} .
$$

Furthermore, Eqs. (B1)-(B3) provide

$$
\begin{aligned}
& \gamma_{u}\left(f_{u}-\sigma_{u u}\right)=R^{\mathrm{ss}}, \gamma_{l}\left(f_{l}-\sigma_{l l}\right)=-R^{\mathrm{ss}}, \\
& \text { and } \quad \gamma_{\mathrm{b}}\left(n_{\mathrm{b}}-n_{\mathrm{ph}}\right)=-R^{\mathrm{ss}} .
\end{aligned}
$$

Inserting into Eq. (A7) (which is the same here), we obtain Eqs. (19). Inserting the relations (B10) and (B11) into the en- ergy flows (B7) and (B8), we find in the steady state Eqs. (20) and (21).

From Eqs. (B6) and (B9), we get $R^{\mathrm{ss}}=\alpha_{Q} F$ with

$$
\alpha_{Q}=\frac{g^{2}\left(\gamma_{u}+\gamma_{l}+\gamma_{\mathrm{b}}\right)}{\Delta_{\mathrm{cav}}^{2}+\left(\gamma_{u}+\gamma_{l}+\gamma_{\mathrm{b}}^{2}\right) / 4}>0
$$

so the sign of $R^{\mathrm{ss}}$ equals the sign of $F$. Using Hartree-Focklike approximations, Eq. (B5) provides

$$
F \approx \sigma_{u u}\left(1-\sigma_{l l}\right)+\left(\sigma_{u u}-\sigma_{l l}\right) n_{\mathrm{ph}} .
$$

Then, straightforward algebra shows

$$
R^{\mathrm{ss}}=\alpha_{Q}\left(1-\sigma_{l l}\right)\left(1-\sigma_{u u}\right)\left(1+n_{\mathrm{ph}}\right)\left(\frac{\sigma_{u u}}{1-\sigma_{u u}}-\frac{\sigma_{l l}}{1-\sigma_{l l}} \times \frac{n_{\mathrm{ph}}}{1+n_{\mathrm{ph}}}\right),
$$

where we assume $f_{u}, f_{l}, \sigma_{u u}, \sigma_{l l}<1$ as appropriate for fermionic states, which are not entirely occupied due to their contribution in a dynamical process. Similarly, Eqs. (B11) can be rewritten as

$$
\begin{gathered}
R^{\mathrm{ss}}=\gamma_{u}\left(f_{u}-\sigma_{u u}\right)=\gamma_{u}\left(1-\sigma_{u u}\right)\left(1-f_{u}\right)\left(\frac{f_{u}}{1-f_{u}}-\frac{\sigma_{u u}}{1-\sigma_{u u}}\right), \\
R^{\mathrm{ss}}=\gamma_{l}\left(\sigma_{l l}-f_{l}\right)=\gamma_{l}\left(1-\sigma_{l l}\right)\left(1-f_{l}\right)\left(\frac{\sigma_{l l}}{1-\sigma_{l l}}-\frac{f_{l}}{1-f_{l}}\right), \\
R^{\mathrm{ss}}=\gamma_{\mathrm{b}}\left(n_{\mathrm{ph}}-n_{\mathrm{b}}\right)=\gamma_{\mathrm{b}}\left(1+n_{\mathrm{ph}}\right)\left(1+n_{\mathrm{b}}\right)\left(\frac{n_{\mathrm{ph}}}{1+n_{\mathrm{ph}}}-\frac{n_{\mathrm{b}}}{1+n_{\mathrm{b}}}\right) .
\end{gathered}
$$

Applying Eqs. (B14)-(B16), subsequently, Eq. (B13) provides

$$
\begin{aligned}
& R^{\mathrm{ss}}=0 \Rightarrow \frac{\sigma_{u u}}{1-\sigma_{u u}}=\frac{f_{u}}{1-f_{u}}, \quad \frac{\sigma_{l l}}{1-\sigma_{l l}}=\frac{f_{l}}{1-f_{l}} \quad \text { and } \frac{n_{\mathrm{ph}}}{1+n_{\mathrm{ph}}}=\frac{n_{\mathrm{b}}}{1+n_{\mathrm{b}}} \Rightarrow \frac{f_{u}}{1-f_{u}}=\frac{f_{l}}{1-f_{l}} \times \frac{n_{\mathrm{b}}}{1+n_{\mathrm{b}}}, \\
& R^{\mathrm{ss}}>0 \Rightarrow \frac{\sigma_{u u}}{1-\sigma_{u u}}<\frac{f_{u}}{1-f_{u}}, \quad \frac{\sigma_{l l}}{1-\sigma_{l l}}>\frac{f_{l}}{1-f_{l}} \quad \text { and } \frac{n_{\mathrm{ph}}}{1+n_{\mathrm{ph}}}>\frac{n_{\mathrm{b}}}{1+n_{\mathrm{b}}} \Rightarrow \frac{f_{u}}{1-f_{u}}>\frac{f_{l}}{1-f_{l}} \times \frac{n_{\mathrm{b}}}{1+n_{\mathrm{b}}}, \\
& R^{\mathrm{ss}}<0 \Rightarrow \frac{\sigma_{u u}}{1-\sigma_{u u}}>\frac{f_{u}}{1-f_{u}}, \quad \frac{\sigma_{l l}}{1-\sigma_{l l}}<\frac{f_{l}}{1-f_{l}} \quad \text { and } \frac{n_{\mathrm{ph}}}{1+n_{\mathrm{ph}}}<\frac{n_{\mathrm{b}}}{1+n_{\mathrm{b}}} \Rightarrow \frac{f_{u}}{1-f_{u}}<\frac{f_{l}}{1-f_{l}} \times \frac{n_{\mathrm{b}}}{1+n_{\mathrm{b}}} .
\end{aligned}
$$

As the right hand-side needs to satisfy one of the three relations, we find equivalence for all relations. This provides the condition (23).

\section{APPENDIX C: GENERALIZATION FOR ARBITRARY SYSTEMS WITH CLASSICAL FIELDS}

The approach for the two-level system used in the main text can be generalized to arbitrary fermionic systems with a time-periodic Hamiltonian $\hat{H}_{S}(t)=\hat{H}_{S}(t+\tau)$ as characteristic for a classical optical field. This includes an arbitrary number of reservoirs, which are coupled to the system in the local form (8), where each reservoir $\alpha$ provides transitions to a unique level $\alpha$ of the system. Here, we assume that the system eventually reaches a steady state $\hat{\rho}^{\mathrm{ss}}(t)$ which is periodic with period $\tau$ following the external driving in $\hat{H}_{S}(t)$ after initial conditions died out due to the dissipative terms in the quantum evolution. We define

$$
\begin{aligned}
\dot{U}_{\alpha}^{\mathrm{av}} & =\left\langle\operatorname{Tr}\left\{\hat{H}_{\mathrm{S}}(t) \mathcal{L}_{\alpha}\left[\hat{\rho}^{\mathrm{ss}}(t)\right]\right\}\right\rangle, \\
\dot{N}_{\alpha}^{\mathrm{av}} & =\left\langle\operatorname{Tr}\left\{\hat{a}_{\alpha}^{\dagger} \hat{a}_{\alpha} \mathcal{L}_{\alpha}\left[\hat{\rho}^{\mathrm{ss}}(t)\right]\right\}\right\rangle,
\end{aligned}
$$

where the averaging

$$
\langle f(t)\rangle=\frac{1}{\tau} \int_{t}^{t+\tau} f\left(t^{\prime}\right) d t^{\prime}
$$

is taken over the common period of $\hat{\rho}^{\mathrm{ss}}(t)$ and $\hat{H}_{\mathrm{S}}(t)$. Thus, $\dot{U}_{\alpha}^{\text {av }}$ and $\dot{N}_{\alpha}^{\text {av }}$ do not depend on time. This allows for the definition of effective energies

$$
\tilde{E}_{\alpha}=\frac{\dot{U}_{\alpha}^{\mathrm{av}}}{N_{\alpha}^{\mathrm{av}}}
$$

which are the average energies taken from reservoir $\alpha$ per particle transferred into the system. It appears natural to apply these energies $\tilde{E}_{\alpha}$ for the energy dependence (16) of the occupation function $f_{\alpha}$ (and possibly also $\gamma_{\alpha}$, if the wide band limit is not applicable). This requires a self-consistent solution for the steady state of the dynamical equation (4) with its input parameters.

In the remaining part of this Appendix, it is shown that this procedure is thermodynamically consistent. The energy is conserved and the entropy production is semipositive for the steady state averaged over a period. This follows concepts used in Ref. [46] for periodically driven systems in a related context.

The general expressions (5) and (6) provide

$$
\frac{d}{d t} \operatorname{Tr}\left\{\hat{H}_{S}(t) \hat{\rho}(t)\right\}=\sum_{\alpha} \dot{U}_{\alpha}+P_{S} .
$$

For the steady state, the average change $\left\langle\frac{d}{d t} \operatorname{Tr}\left\{\hat{H}_{S}(t) \hat{\rho}^{\mathrm{ss}}(t)\right\}\right\rangle$ vanishes due to the integration over one period and we directly get $\sum_{\alpha} \dot{U}_{\alpha}^{\text {av }}+P_{S}^{\text {av }}=0$, showing that the average external 
power and energy currents from the reservoirs going into the system add up to zero. This is just energy conservation for the steady state.

The total entropy $S_{\text {tot }}$ is given by the von Neumann entropy of the system and the reservoirs (changing by heat transfer $\dot{U}_{\alpha}-\mu_{\alpha} \dot{N}_{\alpha}$ into the system), resulting in its temporal change

$$
\frac{d S_{\mathrm{tot}}}{d t}=-k_{B} \frac{d}{d t} \operatorname{Tr}\{\hat{\rho}(t) \ln \hat{\rho}(t)\}-\sum_{\alpha} \frac{\dot{U}_{\alpha}-\mu_{\alpha} \dot{N}_{\alpha}}{T_{\alpha}} .
$$

Upon averaging in the steady state, we get

$$
\dot{S}_{\mathrm{tot}}^{\mathrm{av}}=\left\langle\frac{d S_{\mathrm{tot}}\left[\rho^{\mathrm{ss}}(t)\right]}{d t}\right\rangle=-\sum_{\alpha} \frac{\tilde{E}_{\alpha}-\mu_{\alpha}}{T_{\alpha}} \dot{N}_{\alpha}^{\mathrm{av}} .
$$

Using Eq. (C2), we find

$$
\dot{S}_{\text {tot }}^{\mathrm{av}}=k_{B}\left\langle\sum_{\alpha} \operatorname{Tr}\left\{\mathcal{L}_{\alpha}\left[\hat{\rho}^{\mathrm{ss}}(t)\right] \ln \hat{\rho}^{\mathrm{lt}}\right\}\right\rangle
$$

with the locally thermal operator

$$
\hat{\rho}^{\mathrm{lt}}=\frac{1}{Z^{\mathrm{lt}}} \prod_{\alpha} \exp \left(-\frac{\tilde{E}_{\alpha}-\mu_{\alpha}}{k_{B} T_{\alpha}} \hat{a}_{\alpha}^{\dagger} \hat{a}_{\alpha}\right),
$$

where the number $Z^{\text {lt }}$ renormalizes the trace to unity (and drops out as $\operatorname{Tr}\left\{\mathcal{L}_{\alpha}[\hat{\rho}]\right\}=0$ ). As Eq. (4) gives

$$
\frac{d}{d t} \operatorname{Tr}\{\hat{\rho}(t) \ln \hat{\rho}(t)\}=\sum_{\alpha} \operatorname{Tr}\left\{\mathcal{L}_{\alpha}[\hat{\rho}(t)] \ln \hat{\rho}(t)\right\},
$$

we find for steady-state averaging

$$
0=k_{B}\left\langle\sum_{\alpha} \operatorname{Tr}\left\{\mathcal{L}_{\alpha}\left[\hat{\rho}^{\mathrm{ss}}(t)\right] \ln \hat{\rho}^{\mathrm{ss}}(t)\right\}\right\rangle,
$$

which we can subtract from Eq. (C4), resulting in

$$
\dot{S}_{\text {tot }}^{\mathrm{av}}=k_{B}\left\langle\sum_{\alpha} \operatorname{Tr}\left\{\mathcal{L}_{\alpha}\left[\hat{\rho}^{\mathrm{ss}}(t)\right]\left(\ln \hat{\rho}^{\mathrm{lt}}-\ln \hat{\rho}^{\mathrm{ss}}(t)\right)\right\}\right\rangle .
$$

As $\hat{\rho}^{\text {lt }}$ satisfies $\mathcal{L}_{\alpha}\left[\hat{\rho}^{\mathrm{lt}}\right]=0$ if the occupations (16) are applied, we find $\dot{S}_{\text {tot }}^{\text {av }} \geqslant 0$ by Spohn's inequality $[19,47,48]$. Thus, entropy production is positive semidefinite for the average steady state.
[1] A. E. Siegman, Lasers (University Science Books, MillValley, 1986).

[2] H. Willenberg, G. H. Döhler, and J. Faist, Intersubband gain in a Bloch oscillator and quantum cascade laser, Phys. Rev. B 67, 085315 (2003).

[3] R. Terazzi, T. Gresch, M. Giovannini, N. Hoyler, N. Sekine, and J. Faist, Bloch gain in quantum cascade lasers, Nat. Phys. 3, 329 (2007).

[4] E. Boukobza and D. J. Tannor, Three-Level Systems as Amplifiers and Attenuators: A Thermodynamic Analysis, Phys. Rev. Lett. 98, 240601 (2007).

[5] H. E. D. Scovil and E. O. Schulz-DuBois, Three-Level Masers as Heat Engines, Phys. Rev. Lett. 2, 262 (1959).

[6] I. Roos and K. Mölmer, Quantum computing with an inhomogeneously broadened ensemble of ions: Suppression of errors from detuning variations by specially adapted pulses and coherent population trapping, Phys. Rev. A 69, 022321 (2004).

[7] M. Saffman, T. G. Walker, and K. Mølmer, Quantum information with Rydberg atoms, Rev. Mod. Phys. 82, 2313 (2010).

[8] Thermodynamics in the Quantum Regime: Fundamental Aspects and New Directions, edited by F. Binder, L. A. Correa, C. Gogolin, J. Anders, and G. Adesso (Springer International Publishing, Cham, 2018).

[9] A. Streltsov, G. Adesso, and M. B. Plenio, Colloquium: Quantum coherence as a resource, Rev. Mod. Phys. 89, 041003 (2017).

[10] G. Benenti, G. Casati, K. Saito, and R. S. Whitney, Fundamental aspects of steady-state conversion of heat to work at the nanoscale, Phys. Rep. 694, 1 (2017).

[11] S. Deffner and S. Campbell, Quantum Thermodynamics (Morgan and Claypool, San Rafael, CA, 2019).
[12] A. Levy and R. Kosloff, The local approach to quantum transport may violate the second law of thermodynamics, Europhys. Lett. 107, 20004 (2014).

[13] P. P. Hofer, M. Perarnau-Llobet, L. D. M. Miranda, G. Haack, R. Silva, J. B. Brask, and N. Brunner, Markovian master equations for quantum thermal machines: Local versus global approach, New J. Phys. 19, 123037 (2017).

[14] J. O. González, L. A. Correa, G. Nocerino, J. P. Palao, D. Alonso, and G. Adesso, Testing the validity of the "local" and "global" GKLS master equations on an exactly solvable model, Open Syst. Inf. Dyn. 24, 1740010 (2017).

[15] W. Khan, P. P. Potts, S. Lehmann, C. Thelander, K. A. Dick, P. Samuelsson, and V. F. Maisi, Efficient and continuous microwave photoconversion in hybrid cavity-semiconductor nanowire double quantum dot diodes, Nat. Commun. 12, 5130 (2021).

[16] A. A. S. Kalaee and A. Wacker, Positivity of entropy production for the three-level maser, Phys. Rev. A 103, 012202 (2021).

[17] R. Alicki and R. Kosloff, Introduction to quantum thermodynamics: History and prospects, in Thermodynamics in the Quantum Regime: Fundamental Aspects and New Directions, edited by F. Binder, L. A. Correa, C. Gogolin, J. Anders, and G. Adesso (Springer International Publishing, Cham, 2018), pp. 1-33.

[18] W. Pusz and S. L. Woronowicz, Passive states and KMS states for general quantum systems, Commun. Math. Phys. 58, 273 (1978).

[19] H. Spohn and J. L. Lebowitz, Irreversible thermodynamics for quantum systems weakly coupled to thermal reservoirs, in Advances in Chemical Physics, edited by S. A. Rice (John Wiley \& Sons, New York, 1978), Vol. 38, Chap. 2, pp. 109-142. 
[20] R. Alicki, The quantum open system as a model of the heat engine, J. Phys. A: Math. Gen. 12, L103 (1979).

[21] R. Kosloff and Y. Rezek, The quantum harmonic Otto cycle, Entropy 19, 136 (2017).

[22] J. D. Jackson, Classical Electrodynamics, 3rd ed. (John Wiley \& Sons, New York, 1998).

[23] K. E. Dorfman, K. R. Chapin, C. H. R. Ooi, A. A. Svidzinsky, and M. O. Scully, Quantum thermodynamics of photo and solar cells, AIP Conf. Proc. 1411, 256 (2011).

[24] R. Alicki, D. Gelbwaser-Klimovsky, and A. Jenkins, A thermodynamic cycle for the solar cell, Ann. Phys. 378, 71 (2017).

[25] J. Restrepo, C. Ciuti, and I. Favero, Single-Polariton Optomechanics, Phys. Rev. Lett. 112, 013601 (2014).

[26] E. Geva and R. Kosloff, The quantum heat engine and heat pump: An irreversible thermodynamic analysis of the threelevel amplifier, J. Chem. Phys. 104, 7681 (1996).

[27] K. Szczygielski, D. Gelbwaser-Klimovsky, and R. Alicki, Markovian master equation and thermodynamics of a two-level system in a strong laser field, Phys. Rev. E 87, 012120 (2013).

[28] C. Elouard, D. Herrera-Martí, M. Esposito, and A. Auffèves, Thermodynamics of optical Bloch equations, New J. Phys. 22, 103039 (2020).

[29] R. Hotz and G. Schaller, Coarse-graining master equation for periodically driven systems, Phys. Rev. A 104, 052219 (2021).

[30] G. Lindblad, On the generators of quantum dynamical semigroups, Commun. Math. Phys. 48, 119 (1976).

[31] H.-P. Breuer and F. Petruccione, Open Quantum Systems (Oxford University Press, Oxford, 2006).

[32] J. T. Stockburger and T. Motz, Thermodynamic deficiencies of some simple Lindblad operators, Fortschr. Phys. 65, 1600067 (2017).

[33] C. Bergenfeldt, P. Samuelsson, B. Sothmann, C. Flindt, and M. Büttiker, Hybrid Microwave-Cavity Heat Engine, Phys. Rev. Lett. 112, 076803 (2014).

[34] W. Niedenzu, M. Huber, and E. Boukobza, Concepts of work in autonomous quantum heat engines, Quantum 3, 195 (2019).
[35] E. T. Jaynes and F. W. Cummings, Comparison of quantum and semiclassical radiation theories with application to the beam maser, Proc. IEEE 51, 89 (1963).

[36] P. Santhanam, D. J. Gray, and R. J. Ram, Thermoelectrically Pumped Light-Emitting Diodes Operating above Unity Efficiency, Phys. Rev. Lett. 108, 097403 (2012).

[37] T. Sadi, I. Radevici, and J. Oksanen, Thermophotonic cooling with light-emitting diodes, Nat. Photon. 14, 205 (2020).

[38] L. Mandel and E. Wolf, Optical Coherence and Quantum Optics (Cambridge University Press, Cambridge, 1995).

[39] J. H. Shirley, Validity of the semiclassical approximation in maser theory, Phys. Rev. 181, 600 (1969).

[40] M. Helm, The basic physics of intersubband transitions, in Intersubband Transitions in Quantum Wells, edited by H. Liu and F. Capasso, Semiconductors and Semimetals Vol. 62 (Elsevier, Amsterdam, 1999), pp. 1-99.

[41] S. A. Ktitorov, G. S. Simin, and V. Y. Sindalovskii, Bragg reflections and the high-frequency conductivity of an electronic solid-state plasma, Sov. Phys.-Sol. State 13, 1872 (1972) [Fiz. Tver. Tela 13, 2230 (1971)].

[42] A. Wacker, Semiconductor superlattices: A model system for nonlinear transport, Phys. Rep. 357, 1 (2002).

[43] N. Opačak, S. D. Cin, J. Hillbrand, and B. Schwarz, Frequency Comb Generation by Bloch Gain Induced Giant Kerr Nonlinearity, Phys. Rev. Lett. 127, 093902 (2021).

[44] A. Wacker, Lasers: Coexistence of gain and absorption, Nat. Phys. 3, 298 (2007).

[45] P. P. Potts, A. A. S. Kalaee, and A. Wacker, A thermodynamically consistent markovian master equation beyond the secular approximation, New J. Phys. 23, 123013 (2021).

[46] R. Kosloff, Quantum thermodynamics: A dynamical viewpoint, Entropy 15, 2100 (2013).

[47] H. Spohn, Entropy production for quantum dynamical semigroups, J. Math. Phys. 19, 1227 (1978).

[48] S.-W. Li, Production rate of the system-bath mutual information, Phys. Rev. E 96, 012139 (2017). 